\title{
Substorm onset dynamics in the magnetotail as derived from joint TC-1 and Cluster data analysis
}

\author{
H. Wang ${ }^{1,3}$, H. Lühr ${ }^{2}$, S. Y. Ma ${ }^{1}$, and A. J. Ridley ${ }^{3}$ \\ ${ }^{1}$ College of Electronic Informatics, Wuhan University, Wuhan 430079, P. R. China \\ ${ }^{2}$ GeoForshungsZentrum Potsdam, D-14473 Potsdam, Germany \\ ${ }^{3}$ Department of Atmospheric, Oceanic, and Space Sciences, University of Michigan, Ann Arbor, MI-48109, USA
}

(Received September 9, 2007; Revised February 14, 2008; Accepted February 15, 2008; Online published July 4, 2008)

\begin{abstract}
This study investigates two substorm onset events with favorable constellations of spacecraft, TC-1 and Cluster, separated by several Earth radii. The substorms have been identified in both auroral regions. One is believed to be triggered by a northward turning of the interplanetary magnetic field (IMF), while the other occurs under generally southward IMF. For both events, Cluster was located tailward of TC-1, but observed the dipolarization at earlier time for one event while at later time for the other. The timing difference of dipolarization at different positions could be explained by the earthward or tailward propagation of the field disturbances in the radial direction. The earthward dipolarization front was found in one case to bounce back and forth at TC-1. The earthward propagation was accompanied by a fast earthward plasma flow for the 21 September 2005 event. The event analysis suggests that substorms can be quite different from case to case due to differences in the solar wind condition and magnetotail configuration.
\end{abstract}

Key words: Substorm onset, magnetotail, Cluster, Double Star.

\section{Introduction}

A magnetospheric substorm is a complicated phenomenon that is not fully understood. One of the most controversial topics concerns the exact physical conditions that initiate a substorm. A number of models have reported different onset source locations in the magnetotail. For example, the cross-tail current instability (CCI) model regards the near Earth region $\left(6-10 R_{\mathrm{E}}\right)$ as the initial region (Lui, 1996). It postulates that plasma instabilities generate a cross-tail current disruption and a divergence into the ionosphere via field-aligned currents (FACs) to form the substorm current wedge (SCW). After that, a rarefaction wave is launched tailward, which makes the midtail magnetic configuration more stretched, thus more favorable for reconnection to occur. Therefore, reconnection is started after the substorm onset. Alternatively, the near-Earth neutral line model (NENL) predicts that the substorm is initiated at a further distance down-tail $\left(>20 R_{\mathrm{E}}\right)$, where reconnection takes place (Baker et al., 1996). The formation of the SCW and the associated auroral intensifications are considered as secondary effects of the reconnection. The cause of the SCW can be explained in terms of the braking of the fast earthward bursty bulk flows (Shiokawa et al., 1998) and the pressure gradient driven current induced by the flow braking (Birn et al., 1999). The CCI and NENL models can be distinguished by comparing the time at which reconnection is observed in the tail, utilizing in situ satellite data, with the time when the substorm onset is detected at near-Earth

Copyright (c) The Society of Geomagnetism and Earth, Planetary and Space Sciences (SGEPSS); The Seismological Society of Japan; The Volcanological Society of Japan; The Geodetic Society of Japan; The Japanese Society for Planetary Sciences; TERRAPUB locations. If reconnection is observed first far down-tail, the NENL model is more appropriate (Liou et al., 2002).

During a substorm, dramatic changes take place in the Earth's magnetic field, the magnetospheric and ionospheric currents, and auroral displays, which can be revealed by appropriate measurement techniques. During the past decades, great advances have been achieved in observational techniques for magnetospheric physics. The Double Star mission, TC-1 and TC-2, China's first satellite mission to study the magnetosphere (Liu et al., 2005), combined with the four European Cluster satellites, set up an excellent constellation for exploring Geospace. The magnetic local time of the Double Star at apogee is identical to that of the Cluster spacecraft. In a devoted study, Nakamura et al. (2005) used simultaneous observations of Cluster and Double Star to investigate the relationship between the dipolarization and fast flows. By using multipoint analysis techniques, they determined that the dipolarization front was mainly propagating dawnward. The role of the midtail fast flows in the dissipation process turned out to be quite different, depending on the conditions of the interplanetary magnetic field (IMF). However, detailed ionospheric signatures related to this propagation of the disturbance were not presented. Wang et al. (2006) reported a typical substorm onset event with a fortunate constellation of Cluster and Double Star near the current sheet, which could be one "textbook" example of a substorm due to its distinct character. Both ionosphere and magnetotail observations have shown a clear dawnward propagation of the substorm current wedge. These previous studies have exhibited a current wedge with a large radial extent, which may be another class of substorm that is different from the CCI model and 


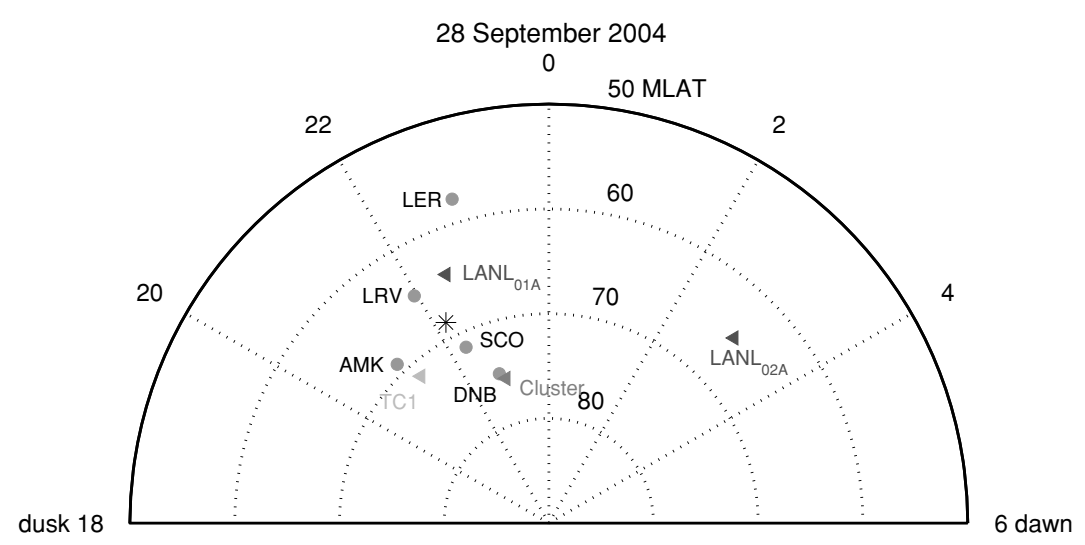

Fig. 1. Conjugate substorm onset region (asterisk), location of TC-1 and Cluster spacecraft (triangle), and magnetometer stations (circle) at auroral breakup time on 28 September 2004.
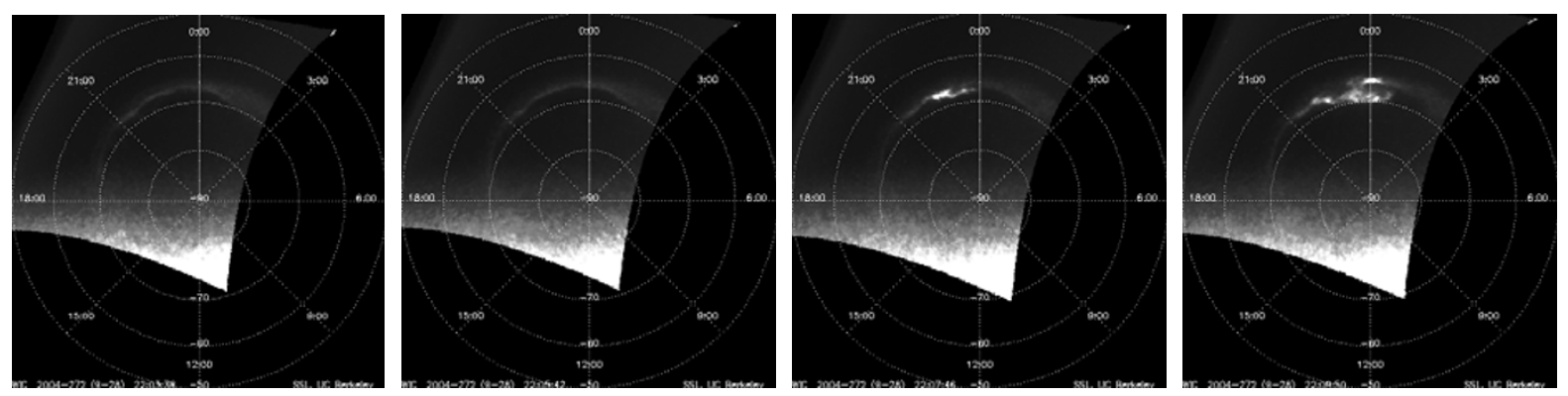

Fig. 2. Auroral imagers from the south polar region taken by IMAGE FUV-WIC at the times 22:03:38, 22:05:42, 22:07:46, and 22:09:50 UT on 28 September 2004. The images are presented in the frame of MLat and MLT coordinates. The sudden bright spot in the middle frame marks the substorm onset.

the NENL model. More events need to be found to confirm the propagation of the substorm disturbances. However, due to the spatially localized nature of substorms in the magnetotail (Nakamura et al., 2005), the number of events with Cluster/TC-1 conjunctions of observations of substorm onset features are quite limited.

In this paper, we present two substorm onset events when both Cluster and TC-1 observed the field dipolarization but at different radial positions. Coordinated observations from geosynchronous satellites, in addition to ground-based magnetometer networks, complement each other quite suitably in the auroral and magnetotail regions where substorm processes are effective.

\section{Observations}

\subsection{The 28 September 2004 event}

The first substorm onset took place at 22:07:46 UT on 28 September 2004 as identified from WIC observations on the IMAGE satellite (Frey and Mende, 2007). About 35 minutes before the onset, a northward turning of the IMF arrived at the magnetopause (the solar wind data are measured by the ACE satellite, which has been time shifted to the magnetopause with the minimum variance method outlined in Weimer et al. (2003)), which might be the interplanetary trigger for the substorm (Lyons, 1996). During the substorm, the magnetic activity was quiet, with a minimum $D_{\mathrm{ST}}$ index of $-6 \mathrm{nT}$, a maximum Kp of $1+$, and a minimum AL of $-246 \mathrm{nT}$.

Figure 1 shows the northern polar ionospheric footpaths of TC-1 and Cluster satellites (triangles), the conjugate location of the substorm auroral breakup (asterisk), and the locations of the considered ground-based magnetometers (circles). At the time of the onset, five magnetometers were found in the vicinity between 21:05 to 22:54 MLT (magnetic local time) ranging from $57.73^{\circ}$ to $74.97^{\circ}$ MLat (magnetic latitude). Cluster-1 was located at $[x, y, z]_{\mathrm{gsm}}=$ $[-14.3,4.3,3.2] R_{\mathrm{E}}$, which mapped to $22: 54 \mathrm{MLT} / 75.6^{\circ}$ MLat, and TC-1 at $[x, y, z]_{\mathrm{gsm}}=[-7.0,6.3,-0.03] R_{\mathrm{E}}$, which mapped to $21: 15$ MLT/71.4 $4^{\circ}$ MLat based on the Tsyganenko magnetic field model (T96). TC-1 was $\sim 7.3 R_{\mathrm{E}}$ earthward and $25^{\circ}$ in longitude duskward of the Cluster quartet.

Figure 2 shows three auroral images of the south polar region from IMAGE FUV-WIC in the frame of MLat and MLT. The time resolution of the aurora observations is 2 minutes. It can be seen that a prominent auroral brightening occurred at $\sim 22: 07: 46$ UT $\left(22: 10\right.$ MLT, $-68.48^{\circ}$ MLat). In the subsequent tens of minutes, the aurora expanded both azimuthally and poleward. These are typical auroral substorm features.

Ground magnetometer recordings can also exhibit typical features of a substorm onset (Lühr et al., 1998). The 1 minute averaged geomagnetic $X, Y$, and $Z$ components measured by five magnetometers during the substorm are shown in Fig. 3. The background magnetic field during quiet time has been subtracted by using monthly median values. The positive variations of the $X$ component at LER seemed to begin at 22:06 UT, which was the onset time in 


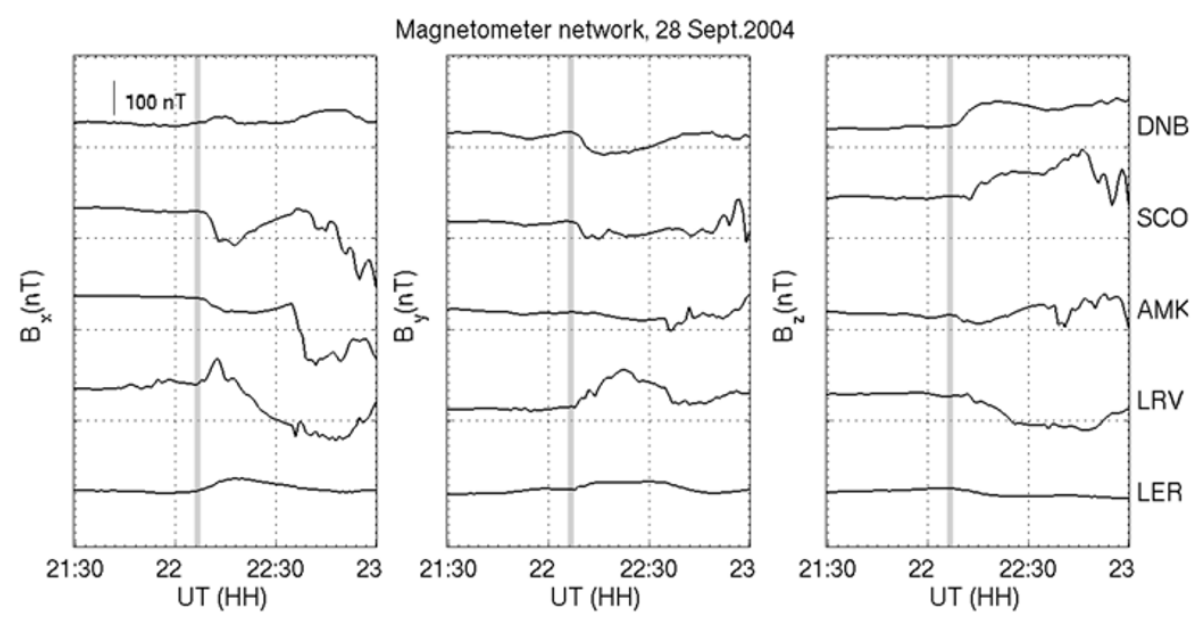

Fig. 3. Magnetograms for the substorm under study, where LRV is from Iceland, LER from United Kingdom, and the remaining three from Greenland. The vertical yellow bar denotes the time of auroral breakup with 2 minutes uncertainty.

the north. This onset time is within the uncertainty range of the IMAGE onset (22:05:46-22:07:46 UT). Therefore, we can assume that the substorm occurred almost simultaneously in both hemispheres. The figure also shows a sharp drop in the $X$ component (positive magnetic northward) at the station SCO (71.44 ${ }^{\circ}$ MLat, 22:19 MLT) after the aurora breakup. This indicates an intense westward electrojet. At LRV (64.8 ${ }^{\circ}$ MLat, 21:57 MLT), after a shortlived positive perturbation in the $X$ component, a major negative magnetic deflection was observed. This is indicative of the passage of a westward travelling surge followed by a westward electrojet. In the surge head an upward FAC is expected (e.g. Kirkwood et al., 1988; Lühr et al., 1998). All this is consistent with the previous CHAMP magnetic field studies (Wang et al., 2005). It can be seen that there was a second sharp drop of the $X$ component after 22:30 UT, which was first registered by the AMK station, followed by the SCO station. The $Z$ component deflection (positive downward) was positive at stations north of LRV and negative south of it, implying an average location of the westward electrojet slightly north of LRV. The IMF $B_{y}$ can cause a hemispheric asymmetry of the MLT of the substorm onset (e.g. Wang $e t$ al., 2007). During the period of the onset we had IMF $B_{y}$ $\sim-4 \mathrm{nT}$. This negative $B_{y}$ will cause the onset in the north to shift to later local time than in the south. Unfortunately, with the limited number of ground stations it is difficult to determine the exact MLT and MLat locations of the north onset. However, the large negative $X$ drop indicates that the center of the westward electrojet is very close to SCO (22:20 MLT), which is a little later than in the south as observed by the IMAGE FUV (22:10 MLT).

Corresponding to this magnetic field activity on the ground caused by a westward electrojet, dispersionless injections at geostationary orbit were observed by the LANL satellites. Figure 4 shows the energetic electron flux data with 10 seconds time resolution from LANL 01A, located in the premidnight sector $(\sim 22: 30$ MLT) at the time of the auroral breakup, and LANL 02A, located postmidnight $(\sim 02: 50 \mathrm{MLT})$ at the time of the auroral breakup. LANL $01 \mathrm{~A}$ detected a significant electron injection at $\sim 22: 11 \mathrm{UT}$, while LANL $02 \mathrm{~A}$ detected it about 1 minute later. An-
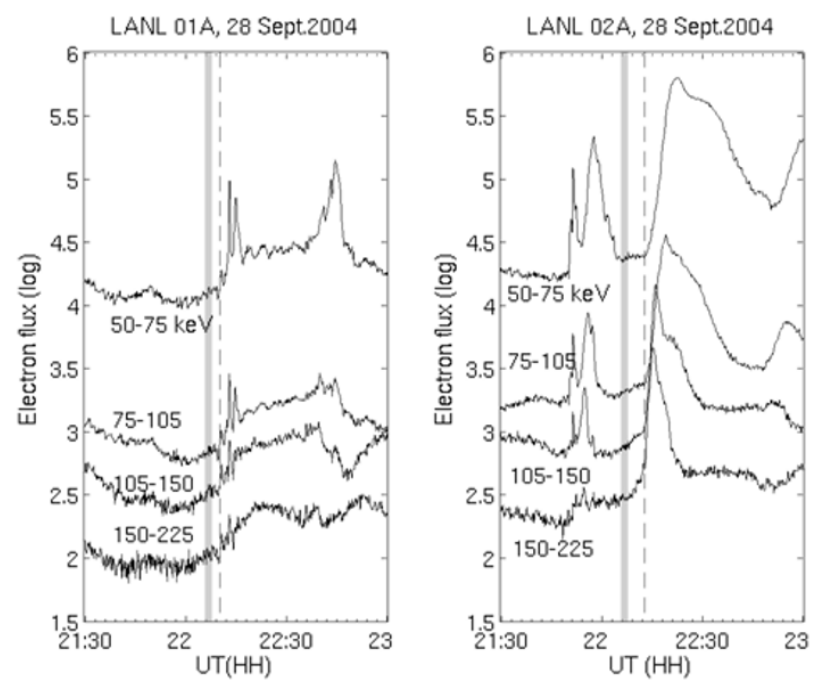

Fig. 4. Differential flux $\left(\mathrm{cm}^{-2} \mathrm{~s}^{-1} \mathrm{sr}^{-1} \mathrm{keV}^{-1}\right)$ of energetic electrons recorded by LANL $01 \mathrm{~A}$ and $02 \mathrm{~A}$, which passed the midnight meridian at 23:30 UT and 19:10 UT, respectively. The energy ranges are shown near the curves. The vertical yellow bar denotes the time of auroral breakup with 2 minutes uncertainty. The vertical dashed green line denotes the time of electron flux injection.

other significant electron injection occurred after 22:30 UT as observed subsequently by these two satellites. Thus, both magnetic ground stations and geosynchronous satellites recorded two onsets of substorm intensification.

Figure 5 shows the magnetotail data in the GSM coordinate system from TC-1 and Cluster between 21:30 and 23:00 UT. The time resolution of the TC- 1 is 6 seconds and that of the Cluster is 10 seconds. TC- 1 was below the current sheet, as can be determined from the negative $B_{x}$, while the Cluster satellites were above the current sheet. For a better localization of the dipolarization of the magnetic field, the elevation angle is shown in Fig. 6. Around 22:06 UT ( 2 minutes before the auroral breakup) Cluster observed a clear magnetic dipolarization. There were some wave like oscillations in the TC-1 magnetic field around 22:04 UT, which might be identified as the onset time of the dipolarization at TC-1. This wavelike oscillation observed at TC-1 
Table 1. Time of substorm disturbances.

\begin{tabular}{cccc}
\hline UT time & 28 September 2004 & UT time & 21 September 2005 \\
\hline $22: 04$ & Onset of field dipolarization at TC-1 & $13: 59$ & Onset of field dipolarization at Cluster \\
$22: 06$ & Onset of field dipolarization at Cluster & $14: 04$ & Onset of field dipolarization at TC-1 \\
22:05:46-22:07:46 & Auroral breakup observed by IMAGE & $14: 03: 55-14: 05: 55$ & Auroral breakup observed by IMAGE \\
$22: 06$ & Onset of ground magnetic disturbances at LER & $14: 06-14: 22$ UT & Onset of ground magnetic disturbances \\
$22: 11$ & Injection at LANL01A & $14: 06$ & A small injection at LANL 1994-084 \\
$22: 12$ & Injection at LANL02A & - & - \\
\hline
\end{tabular}
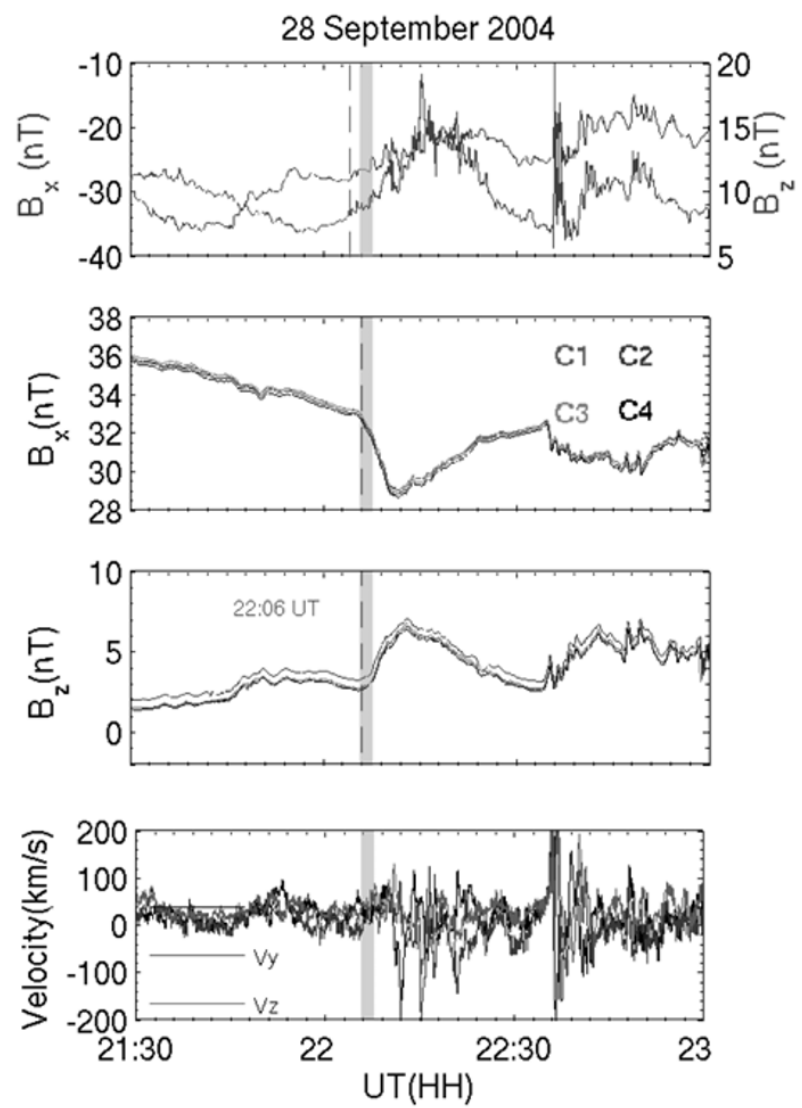

Fig. 5. Magnetotail observations of TC-1 and Cluster. In the top three panels are shown $B_{x}, B_{z}$ in GSM coordinates observed by TC-1, and by the Cluster quartet. In the bottom panel is proton velocity observed by TC-1 HIA (Hot ion analyser). The vertical yellow bar denotes the time of auroral breakup with 2 minutes uncertainty, red and green dashed line, the time of field dipolarization at TC- 1 and Cluster.

might indicate that current instabilities were initiated in the inner region then propagating tailward. Around onset, no significant plasma flow was detected (see Fig. 5 bottom). However, for the general features of the field dipolarization, there was a rather similar overall variation of the elevation angle at TC-1 and Cluster. A cross-correlation analysis was performed over the period from 22:00 to 22:30 UT in order to determine the lag time between TC-1 and Cluster. Figure 7 shows the correlation coefficients as a function of lag time between TC-1 and the four Cluster satellites. The lag time indicates that on average, for the overall dipolarization feature, TC- 1 responded about 2 minutes delayed with respect to Cluster. It should be noticed that both Cluster and TC-1 observed another field dipolarization shortly after 22:30 UT (see Fig. 6). It is clear in this case that
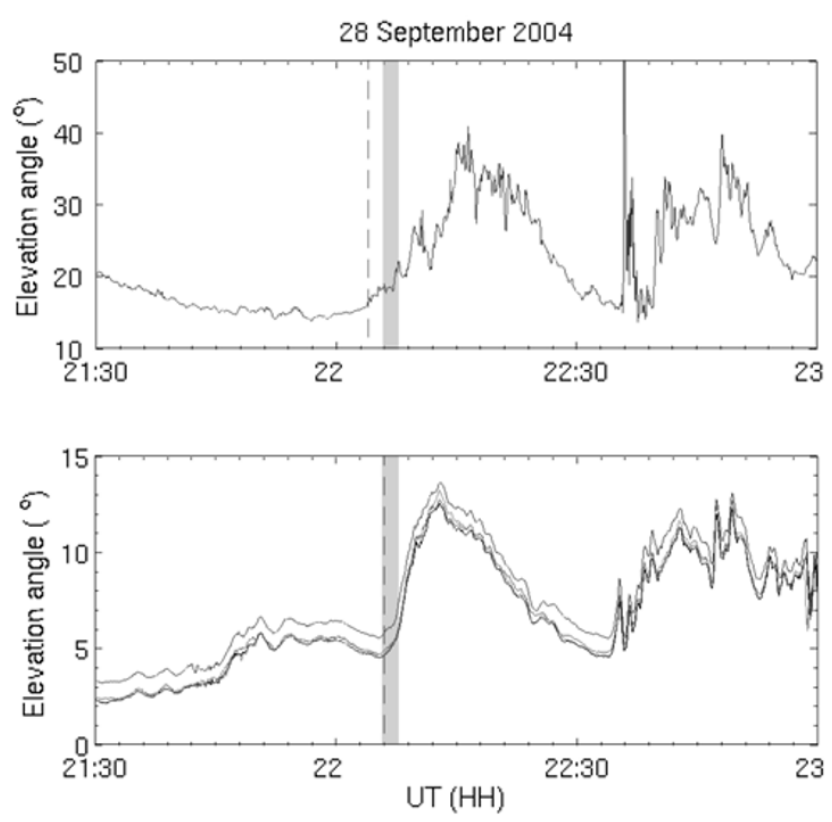

Fig. 6. In the top panel are shown the elevation angles of the magnetic field in GSM coordinates observed by TC-1, below by Cluster quartet. The vertical yellow bar denotes the time of auroral breakup with 2 minutes uncertainty, red and green dashed line, the time of field dipolarization at TC-1 and Cluster.

TC-1 observed the onset of the disturbance after Cluster. The signature at TC-1 was intermittent. This means that the dipolarization front was first moving earthward past Cluster and TC-1, then retreated tailward for a while past TC-1 and finally moved earthward again where it remained. This feature can be confirmed by the $B_{y}$ variations, which will be discussed further in Section 3. From the above analysis the time of the first initialization can be determined. The start of this initial substorm activity was observed first at TC-1, then Cluster, then ground magnetometer, auroral imagers, then finally at LANL. Dipolarization at TC-1 occurred earlier than at Cluster indicating that the substorm is initiated in the near-earth region. The fact that TC-1 observed the overall dipolarization features later than Cluster indicates that after the near-earth initiation the far tail reconnection occurred, which propagated the large scale disturbances earthward, from Cluster to TC-1. The time line of activity for this substorm around 22:07:46 UT is listed in Table 1.

\subsection{The 21 September 2005 event}

The second typical substorm onset presented here took place at 14:05:55 UT (01:42 MLT, $-67.6^{\circ}$ MLat) on 21 September 2005 as identified by IMAGE WIC auroral ob- 


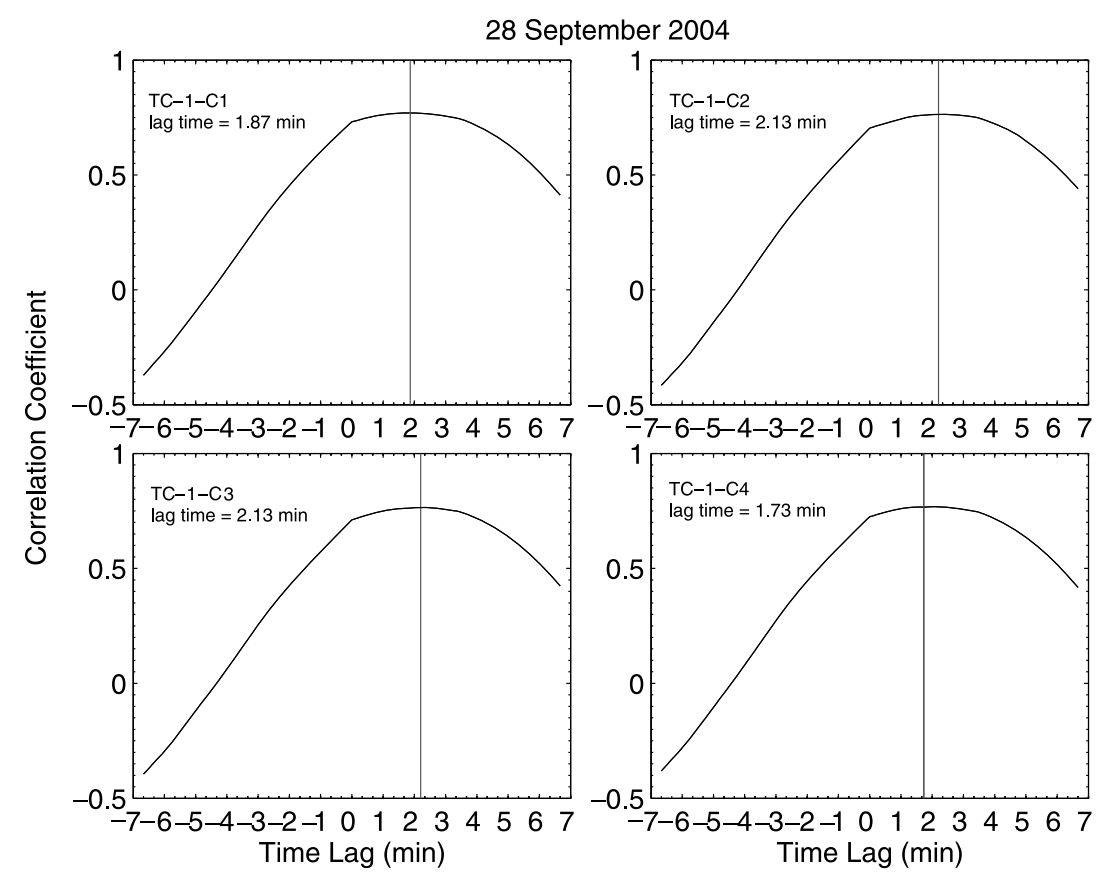

Fig. 7. Cross correlation of the variation of the elevation angle between TC-1 and those four Cluster satellites during the period from 22:00 to 22:30 UT. The vertical blue line denotes the lag time when the correlation coefficient reaches maximum.

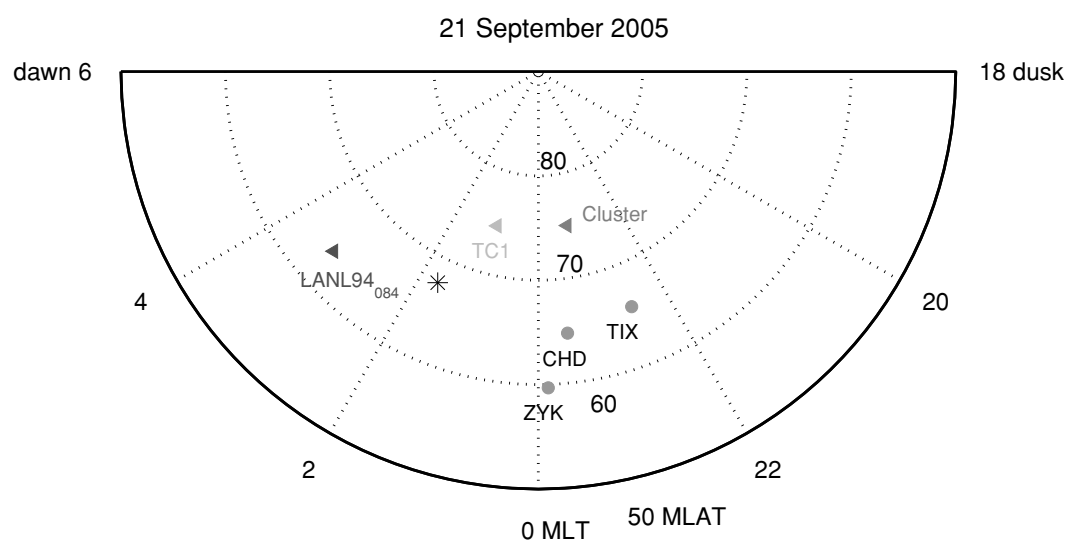

Fig. 8. Conjugate substorm onset region (asterisk), location of TC-1 and Cluster spacecraft (triangle), and $210 \mathrm{MM}$ magnetometer stations (circle) at auroral breakup time on 21 September 2005.

servations (Frey and Mende, 2007). One hour before the substorm onset, IMF $B_{z}$ had an almost constant magnitude of -2 nT. Positive IMF $B_{x}$ and $B_{y}$ underwent minor variations (less than $2 \mathrm{nT}$ ). The solar wind dynamic pressure stayed roughly constant. During the substorm the magnetic activity was normal, with a minimum $D_{\mathrm{ST}}$ index of $-27 \mathrm{nT}$, a maximum $\mathrm{Kp}$ of 2 , and a minimum $\mathrm{AL}$ of $-268 \mathrm{nT}$.

Figure 8 shows the northern polar ionospheric footpaths of the Cluster and TC-1 satellites, the conjugate location of the substorm auroral breakup (black asterisk), as identified by IMAGE-WIC, and the position of ground-based magnetometers $\left(210^{\circ}\right.$ Magnetic Meridian chain). At the time of onset (14:06 UT), recordings from three magnetometers were available, located around 22:30-23:53 MLT ranging from $59.68^{\circ}$ to $65.75^{\circ}$ MLat. Cluster- 1 was located at $[x, y, z]_{\mathrm{gsm}}=[-15.96,1.78,1.81] R_{\mathrm{E}}$, which mapped to 23:18 MLT $/ 75^{\circ}$ MLat. TC-1 was at $[x, y, z]_{\mathrm{gsm}}=[-11.15$, $-5.6,3.35] R_{\mathrm{E}}$, which mapped to 01:00 MLT/74.7 $7^{\circ}$ MLat.
TC-1 was displaced $\sim 4.8 R_{\mathrm{E}}$ earthward and $33^{\circ}$ in longitude dawnward from the Cluster quartet.

Figure 9 shows three auroral images just 2 minutes before, at, and after the substorm onset. The auroral breakup was reported to occur at 14:05:55 UT in the postmidnight sector. It should be noted that there was another auroral brightening in the premidnight sector, which originated at 13:30 UT (auroral images are not shown). This premidnight auroral breakup diminished around 14:08 UT. At the time of the auroral breakup, the three magnetometers shown in Fig. 9 were about 2 hours away from the conjugate footprint region of the auroral breakup. During the period of the onset, the IMF $B_{y}$ is $\sim 4 \mathrm{nT}$. The positive $B_{y}$ will cause the onset in the north to shift to earlier local time than in the south, thus bringing it closer to the magnetometers. The magnetic variations of the $X, Y$, and $Z$ components, with 1 minute time resolution throughout the substorm, are shown in Fig. 10. Both CHD and TIX recorded a drop in 

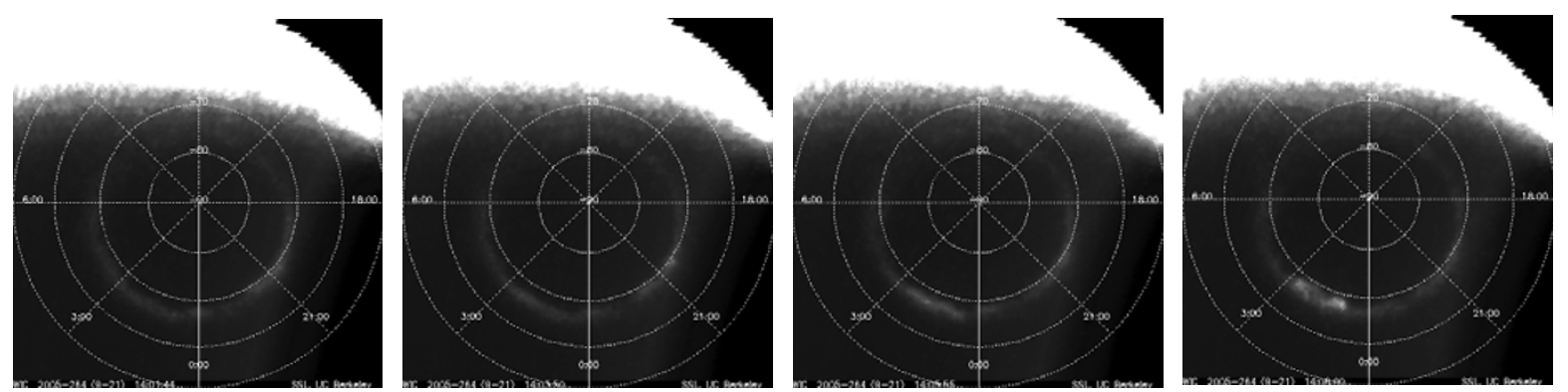

Fig. 9. Auroral imagers from the south polar region taken by IMAGE FUV-WIC at the times 14:01:44, 14:03:50, 14:05:55, and 14:08:00 UT on 21 September 2005. The images are presented in the frame of MLat and MLT coordinates. The auroral breakup is reported to occur at 14:05:55 UT.

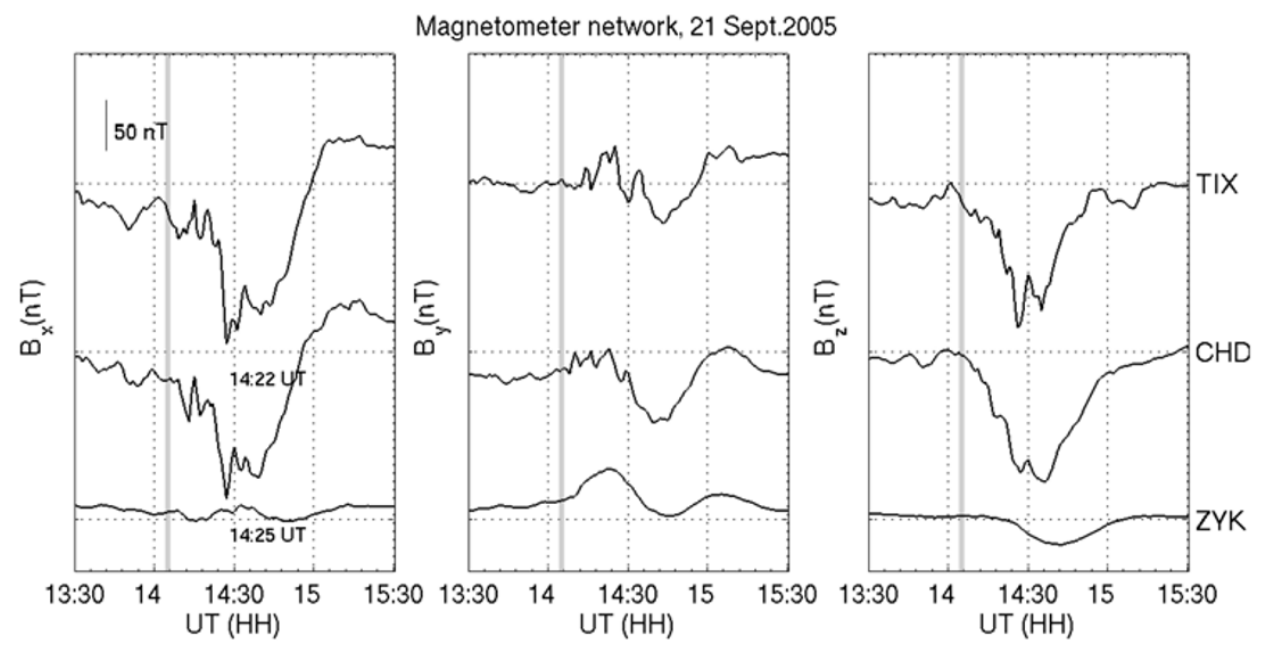

Fig. 10. Magnetograms from the $210^{\circ}$ Magnetic Meridian for the substorm under study. The vertical yellow bar denotes the time of auroral breakup with 2 minutes uncertainty.

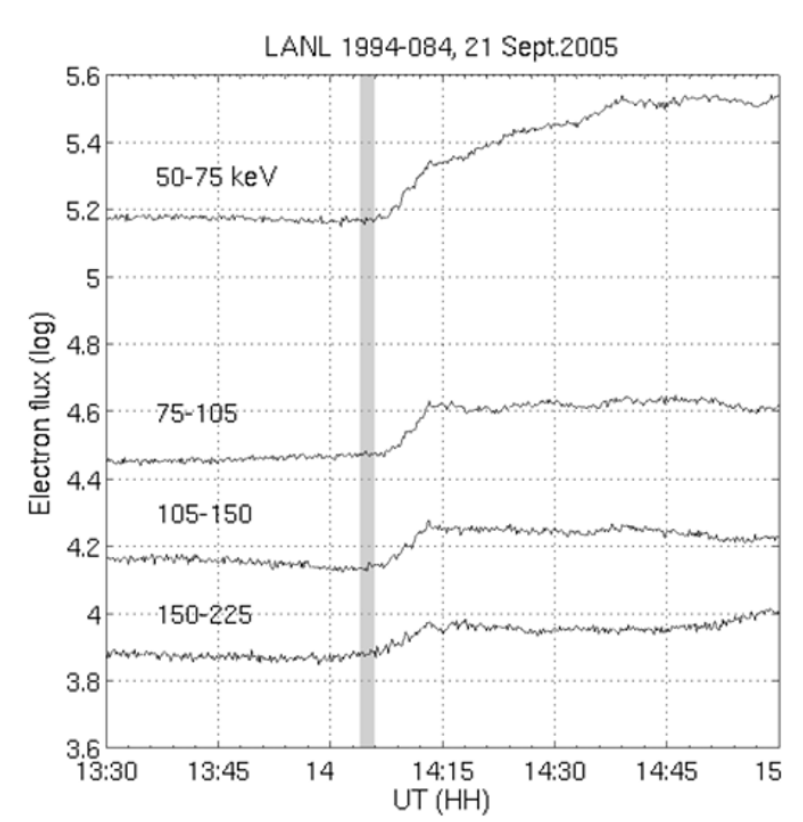

Fig. 11. Differential flux $\left(\mathrm{cm}^{-2} \mathrm{~s}^{-1} \mathrm{sr}^{-1} \mathrm{keV}^{-1}\right)$ of energetic electrons recorded by LANL 1994-084, which passed the midnight meridian at 11:00 UT. The energy ranges are shown near the curves. The vertical yellow bar denotes the time of auroral breakup with 2 minutes uncertainty. the $X$ component, which started around 13:30 UT. This was associated with the premidnight auroral breakup. Following that there was a two step drop. The first slow drop was still associated with the premidnight substorm auroral breakup. The second rapid drop with much larger magnetic deflections (14:22 UT at CHD and 14:25 UT at TIX), was associated with the substorm electrojet under study. But the onset times of the ground magnetic disturbance associated with the substorm around 14:06 UT were not clear due to the overlapping substorm activity. A remarkable feature in the $Y$ component at all three stations was a sinusoidal variation (see Fig. 10). It drifted westward and equatorward over the stations. We can determine a westward propagation velocity for the current structures of $\sim 0.08^{\circ} / \mathrm{s}(1.28 \mathrm{~km} / \mathrm{s})$ from the signal delay between TIX and CHD using a cross correlation analysis. We can also determine the equatorward shift velocity to be $\sim 0.04 \% \mathrm{~s}(0.64 \mathrm{~km} / \mathrm{s})$ from CHD and ZYK. The $Z$ component deflection (positive downwards) was negative at all the stations implying a location of the electrojet polewards of the magnetometer stations. The observations clearly show that this substorm also occurred in both hemispheres. Corresponding to the recorded auroral and ground based signatures, a small but significant electron injection was observed by LANL 1994-084 at 14:06 UT in the postmidnight sector around 03:00 MLT (see Fig. 11). But no electron injections were observed associated with the premidnight auroral breakup around 13:30 UT. 

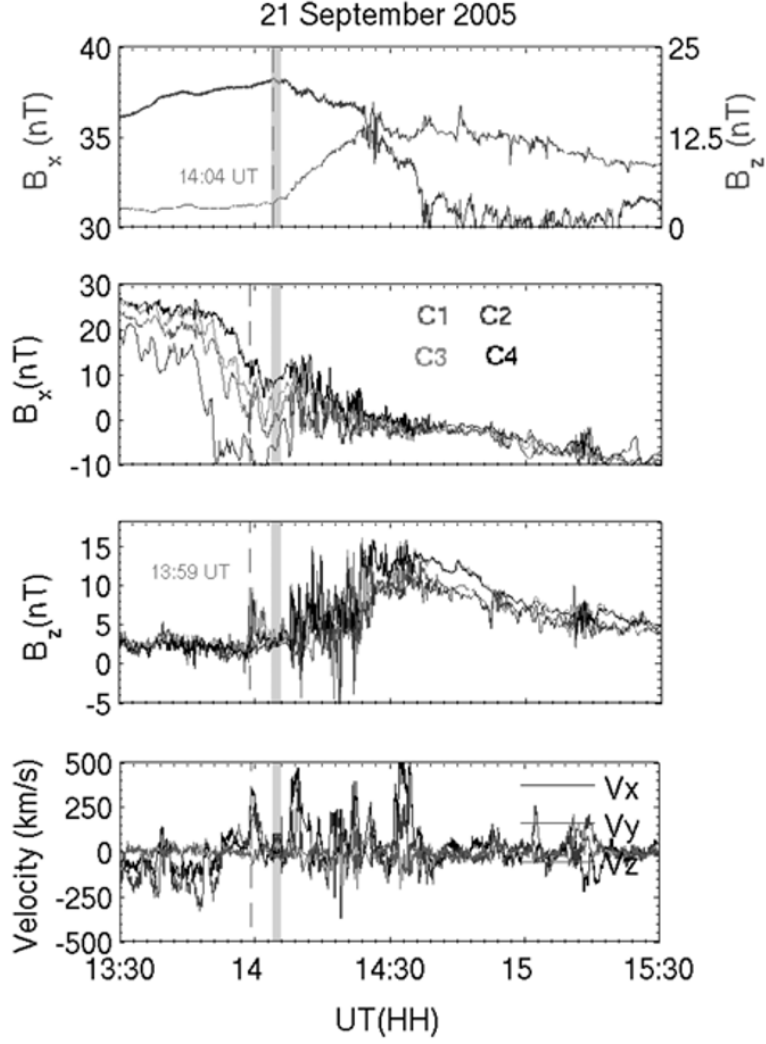

Fig. 12. Magnetotail observations of TC-1 and Cluster. In the top three panels are shown $B_{x}, B_{z}$ in GSM coordinates observed by TC-1, and the Cluster quartet. The bottom panel shows the velocity of hot ion observed by Cluster HIA. The vertical yellow bar denotes the time of auroral breakup with 2 minutes uncertainty, red and green dashed line, the time of field dipolarization at TC-1 and Cluster.

Figure 12 shows magnetotail data in the GSM coordinate system from TC-1 and Cluster between 13:30 and 15:30 UT. TC-1 and the Cluster satellites are above the current sheet at the start of the time period, as could be deduced from the positive $B_{x}$ component. Details of the dipolarization are presented in Fig. 13, where elevation angles of the magnetic field are shown. At 14:04 UT, TC-1 observed a clear magnetic dipolarization. The Cluster spacecraft measured a $B_{x}$ decrease $\sim 15$ minutes before the auroral breakup, while they observed only small fluctuations in the $B_{z}$ component (see Fig. 12). This is an indication that the quartet approached and partly crossed the cross-tail current sheet. $B_{z}$ began to increase about 7 minutes before the auroral breakup, although somewhat intermittently. When looking at the elevation angle in Fig. 13, it is possible to estimate the onset of field dipolarization at Cluster to be 13:59 UT, which was 7 minutes before the auroral breakup. The field dipolarization was found to be associated with fast earthward busty bulk flows (see Fig. 12 bottom). Neither TC-1 nor Cluster observed field disturbances associated with the premidnight auroral breakup around 13:30 UT, indicating a psuedobreakup event. From the above analysis, the time sequence of the substorm activity around 14:06 UT was first Cluster, then TC-1 and LANL. As mentioned earlier, the onset time of ground based measurements is not clear due to the repeated auroral breakups. The time line of activity for this substorm is also listed in Table 1.
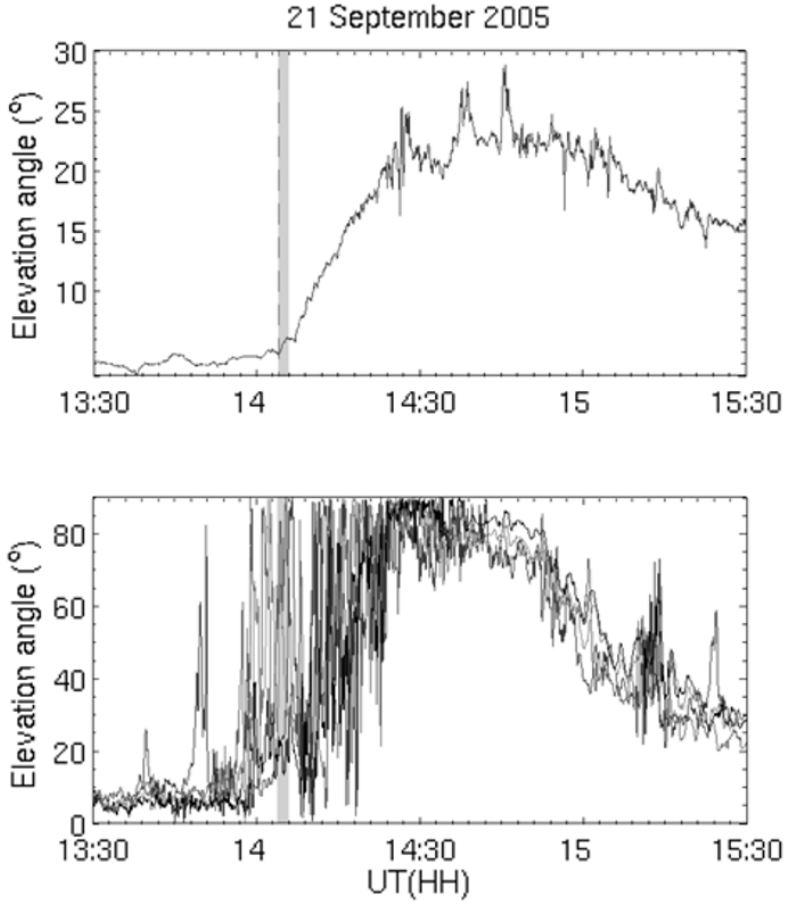

Fig. 13. Top panel shows the elevation angle of magnetic field in GSM coordinates observed by TC-1, below by Cluster quartet. The vertical yellow bar denotes the time of auroral breakup with 2 minutes uncertainty and the red line, start of dipolarization at TC-1, and green, $\sim 7$ minutes ealier, the time of field dipolarization at Cluster.

\section{Discussion and Conclusion}

The observed characteristics in both the auroral zone and magnetotail region during two well developed substorms have been presented. The magnetic fields, the geosynchronous plasma distribution, ionospheric currents, and auroral displays have shown rapid changes. TC-1 and Cluster were not only separated in the radial direction but also in the longitudinal direction, i.e., Cluster was eastward $\left(\sim 2 R_{\mathrm{E}}\right.$ or $25^{\circ}$ in long.) for the 28 September 2004 event, while westward ( $\sim 7 R_{\mathrm{E}}$ or $33^{\circ}$ in long.) for the 21 September 2005 event. For the 2004 substorm event, TC-1 observed the onset of the field dipolarization earlier than Cluster, but observed the general dipolarization feature later than Cluster. For the 2005 substorm events, Cluster observed the field dipolarization earlier than TC-1.

For the 28 September 2004 event, TC-1 observed some field disturbances $\sim 2$ minutes earlier than Cluster, indicating that current instabilities were initiated in the inner region then propagating tailward. For the general field dipolarization feature, TC- 1 is clearly $\sim 2$ minutes later than Cluster, indicating an earthward expansion of the large scale field disturbances. This large scale field disturbance might be caused by reconnection in the far tail, which occurred later than the current disruption in the inner region. To understand the front propagation of field disturbances in a relatively simple way, satellites have been projected to the ground for comparison, as shown in Fig. 1. If the time sequence was dominated by the radial distance, starting from the inner earth region, the expected time sequence would be TC-1 to Cluster. The subsequent far-tail reconnection propagated the disturbances further earthward, that is, from 

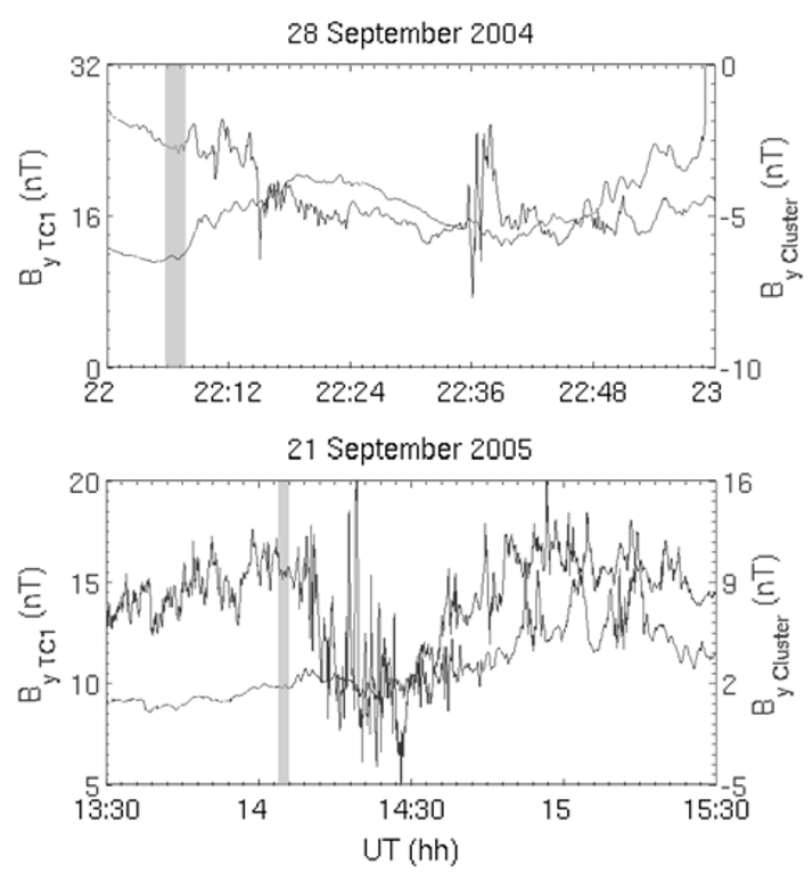

Fig. 14. TC-1 and Cluster observation of the $B_{y}$ components in GSM coordinates on 28 September 2004 (top) and on 21 September 2005 (bottom). The vertical yellow bar denotes the time of auroral breakup with 2 minutes uncertainty.

Cluster to TC-1 to LANL-01A. Therefore, we may conclude that a temporary tailward propagation of the field disturbance front can be observed, but this is embedded in an overall earthward expanding dipolarization front.

For the 21 September 2005 event, a timing difference of 7 minutes in the magnetic dipolarization signatures at TC- 1 and Cluster could also be associated with the earthward propagation of the field disturbances. Figure 8 shows the distribution of satellites and magnetometers. The succession of activation was Cluster-TC-1-LANL. On the first glimpse, it seemed that either dawnward or earthward propagation could explain the sequences of events. However, the dawnward propagation was inconsistent with the substorm disturbance spreading from the postmidnight to the premidnight sector, based on WIC images and the time sequence of ground magnetic activities. Therefore, the substorm activity for this event showed a propagation of the field disturbances from the high latitude, i.e., the mid-tail region, to the low latitude, i.e., the near-Earth region.

Besides determining the propagation of the dipolarization front, we have examined to which part of the current wedge the various spacecraft were connected. For this purpose, the field-aligned currents flowing at the time of the substorm onset were examined. Figure 14 shows the TC-1 and Cluster variations of the $B_{y}$ components in GSM coordinates on 28 September 2004 (top) and 21 September 2005 (bottom). Previous studies have shown that there is a dominant upward FAC in the vicinity of the onset location, which is believed to be associated with the auroral breakup (e.g. Lyons et al., 2003; Wang et al., 2005). When mapped into the magnetosphere, this upward FAC at the duskward end of the current wedge should flow tailward in both hemispheres. As mentioned in Section 2.1, for the 28 Septem-
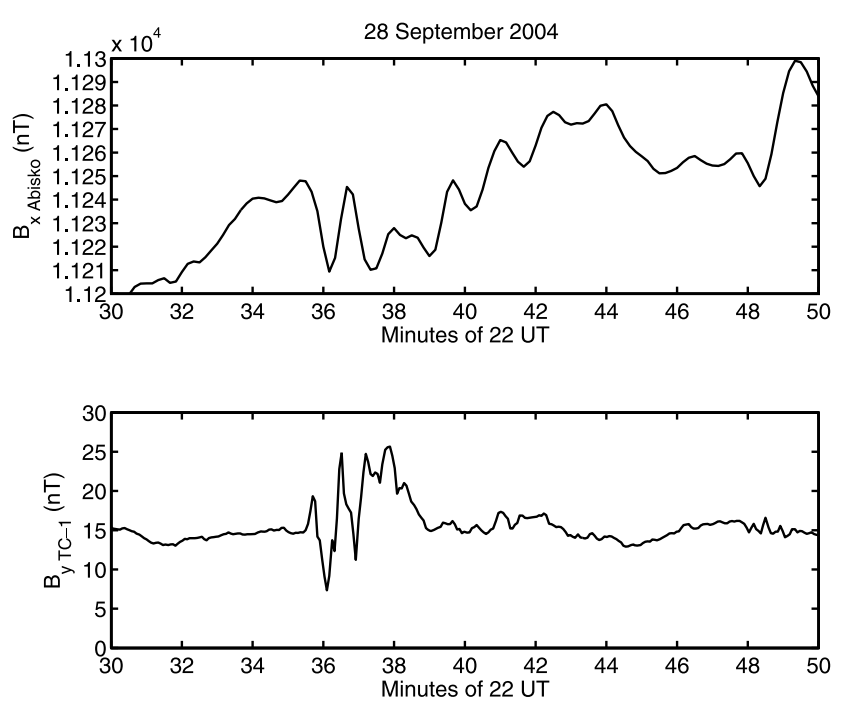

Fig. 15. Ground and space-based observations of a Pi2 pulsation. The upper curve shows the magnetic field variations of the northward component at Abisko and the lower the $B_{y}$ component variations at TC- 1 .

ber 2004 event, TC-1 and Cluster were located on opposite sides of the current sheet. As seen in Fig. 14, right after the time of auroral breakup, Cluster sensed a steep positive gradient in the $B_{y}$ component. This could be interpreted as the earthward crossing of a major upward FAC sheet. TC-1 recorded some fluctuations in $B_{y}$ before and after the onset, but about 7 minutes later a step-like decrease occurred. This is also indicative of a fast earthward passage of an upward FAC sheet. Consequently, both spacecraft sensed predominantly tailward flowing FACs but at different times. TC-1 measured further large variation in $B_{y}$ after 22:35 UT. These were well phased with the second field dipolarization, as mentioned in Section 2.1 (see Fig. 6). Simultaneously, with the shortlived dipolarization at TC-1 (22:35-22:37 UT), the $B_{y}$ component showed large oscillations. These seem to be the magnetospheric signature of a Pi2 pulsation. An inspection of magnetograms on the nightside revealed that there was actually a $\mathrm{Pi} 2$ event at that time. Since it is a very rare case to have a satellite observing directly the field-aligned currents associated with a Pi2, we show in Fig. 15 the phase relation between the groundbased recordings at Abisko (65.1 ${ }^{\circ}$ MLat, 00:09 MLT) and the TC-1 $B_{y}$ component on an expanded scale. At 22:37 UT, the dipolarization front retreated tailward past TC- 1 . The positive deflection of $B_{y}$ was consistent with an upward FAC sheet tailward of the spacecraft. Only 2 minutes later, the dipolarization front swept back earthward, accompanied by a steep negative gradient in $B_{y}$. This indicated a final earthward passage of the upward FAC. In summary, TC-1 had the fortunate opportunity to observe an earthward propagating dipolarization front that reversed its direction for a short while before it finally passed earthward. We may conclude that temporary tailward propagations of the current disruption front can be observed, but these are still embedded in an overall earthward expansion. As seen in Fig. 14, on 21 September 2005, the Cluster $B_{y}$ component went negative from 14:06 UT onward, suggesting an earthward passage of a downward FAC. The TC- $1 B_{y}$ followed 
the trend at Cluster with a time delay, again implying the earthward passage of the FAC sheet. After 14:25 UT the $B_{y}$ component at TC-1 was steeply rising. This may be interpreted as a duskward propagation of the current wedge center line passed TC-1.

Our initial aim was to make use of the multi-spacecraft configuration to find support for any of the competing substorm onset models. This could not be achieved conclusively by these two case studies. However, some preferences for one of the models may be deduced. One event showed that TC-1 observed field turbulence earlier than the Cluster quartet, thus, supporting the CCI model. However, the other event showed that TC-1 observed field dipolarization later than the Cluster quartet, thus, supporting the NENL model. Both events suggest the importance of the reconnection in the far tail with the large-scale activity propagating from the midtail to the inner magnetosphere. Even though this study focused mainly on dipolarization in the magnetotail, associated parameters such as plasma flow in the magnetotail were also considered in differentiating between the two substorm models (see Figs. 5 and 12). For the 21 September 2005 event, the earthward expansion of the field dipolarization was found to be associated with fast earthward busty bulk flows (see Fig. 12). This fast earthward flow together with a positive $B_{z}$ was believed to be related to an earthward moving flux rope type signature (Nakamura et al., 2005). However, no significant plasma flow was detected during the 28 September 2004 event (see Fig. 5), which can be explained by the fact that the spacecraft were not located in the central plasma sheet. To further test the casual relationship of substorm onsets more spacecraft in radial constellation near the current sheet like THEMIS are needed.

Acknowledgments. We thank Double Star/Cluster, IMAGE, and LANL teams for satellite operation and data processing. Greenland, LRV, LER, and 210 MM Magnetic Observation Project teams are also greatly acknowledged for providing magnetic field data. We also thank ACE teams for providing solar wind and IMF data, and WDC C2 for Geomagnetism at Kyoto for providing geomagnetic indices data. This study is supported by the NASA research grant F010639, NSF grant ATM0639336, and National Nature Science Foundation of China No. 40604017.

\section{References}

Baker, D. N., T. I. Pulkkinen, V. Angelopoulos, W. Baumjohann, and R. L. McPherron, Neutral line model of substorms: Past results and present view, J. Geophys. Res., 101, 12,975-13,010, 1996.
Birn, J., M. Hesse, G. Haerendel, W. Baumjohann, and K. Shiokawa, Flow braking and the substorm current wedge, J. Geophys. Res., 104, 19,89519,904, 1999.

Frey, H. U. and S. B. Mende, Substorm onset observations by IMAGEFUV: 2003-2005, in Proceedings of the Eighth International Conference on Substorms, edited by M. Syrjäsuo and E. Donovan, 71-75 pp, Univ. of Calgary, Alberta, Can., 2007.

Kirkwood, S., H. J. Opgenoorth, and J. S. Murphree, Ionospheric conductivities, electric fields and currents associated with auroral substorms measured by the EISCAT radar, Planet. Space. Sci., 36, 1359-1380, 1988.

Liou, K., C. I. Meng, A. T. Y. Lui, P. T. Newell, and S. Wing, Magnetic dipolarization with substorm expansion onset, J. Geophys. Res., 107, doi:10.1029/2001JA000179, 2002.

Liu, Z. X., C. P. Escoubet, Y. Z. Pu, H. Laakso, J. K. Shi, C. Shen, and M. Hapgood, The double star mission, Ann. Geophys., 23, 2707-2712, 2005.

Lühr, H., A. Aylward, S. C. Bucher, A. Pajunpaeae, K. Pajunpää, T. Holmboe, and S. M. Zalewski, Westward moving dynamic substorm features observed with the IMAGE magnetometer network and other groundbased instruments, Ann. Geophys., 16, 425-440, 1998.

Lui, A. T. Y., Current disruption in the Earth's magnetosphere: Observations and models, J. Geophys. Res., 101, 13,067-13,088, 1996.

Lyons, L. R., Substorms: Fundamental observational features, distinction from other disturbances, and external triggering, J. Geophys. Res., 101, 13,011-13,026, 1996.

Lyons, L. R., C.-P. Wang, and T. Nagai, Substorm onset by plasma sheet divergence, J. Geophys. Res., 108, doi:10.1029/2003JA010,178, 2003.

Nakamura, R., W. Baumjohann, T. L. Zhang, C. M. Carr, A. Balogh, K.-H. Fornacon, E. Georgescu, H. Réme, I. Dandouras, T. Takada, M. Volwerk, Y. Asano, A. Runov, H. Eichelberger, B. Klecker, C. Mouikis, L. M. Kistler, and O. Amm, Cluster and Double Star observations of dipolarization, Ann. Geophys., 23, 2915-2920, 2005.

Shiokawa, K., W. Baumjohann, G. Haerendel, G. Paschmann, J. F. Fennell, E. Friis-Christensen, H. Lühr, G. D. Reeves, C. T. Russel, P. R. Sutcliffe, and K. Takahashi, High-speed ion flow, substorm current wedge, and multiple Pi2 pulsations, J. Geophys. Res., 103, 4491-4507, 1998.

Wang, H., H. Lühr, S. Y. Ma, and P. Ritter, Statistical study of the substorm onset: its dependence on solar wind parameters and solar illumination, Ann. Geophys., 23, 2069-2079, 2005.

Wang, H., S. Y. Ma, H. Lühr Z. X. Liu, Z. Y. Pu, C. P. Escoubet, H. U. Frey, H. Réme, and P. Ritter, Global manifestations of a substorm onset observed by a multi-satellite and ground station network, Ann. Geophys., 24, 3491-3496, 2006.

Wang, H., H. Lühr, S. Y. Ma, H. U. Frey, Interhemispheric comparison of average substorm onset locations: Evidence for deviation from conjugacy, Ann. Geophys., 25, 989-999, 2007.

Weimer, D. R., D. M. Ober, N. C. Maynard, M. R. Collier, D. J. McComas, N. F. Ness, C. W. Smith, and J. Watermann, Predicting interplanetary magnetic field (IMF) propagation delay times using the minimum variance technique, J. Geophys. Res., 108, doi:10.1029/2002JA009,405, 2003.

H. Wang (e-mail: whui@umich.edu), H. Lühr, S. Y. Ma, and A. J. Ridley 